\title{
Reliability of Decision-Making and Reinforcement Learning
}

$5 \quad{ }^{1}$ Neuroscience and Mental Health Group, Institute of Cognitive Neuroscience, University

6 College London, London, United Kingdom.

$8 *$ Correspondence

$9 \quad$ Anahit Mkrtchian

10 Institute of Cognitive Neuroscience, University College London

11 Alexandra House, 17-19 Queen Square

12 London, WC1N 3AZ

13 United Kingdom

14 Email: a.mkrtchian@ucl.ac.uk

15

16 Running Title: Reliability of computational parameters

17

18 Keywords: Reliability, Computational psychiatry, Decision-making, Reinforcement learning,

19 Prospect theory, Gambling 


\section{Abstract}

2 Computational models can offer mechanistic insight into cognition and therefore have the

3 potential to transform our understanding of psychiatric disorders and their treatment. For

4 translational efforts to be successful, it is imperative that computational measures capture

5 individual characteristics reliably. To date, this issue has received little consideration. Here we

6 examine the reliability of reinforcement learning and economic models derived from two

7 commonly used tasks. Healthy individuals $(\mathrm{N}=50)$ completed a restless four-armed bandit and a

8 calibrated gambling task twice, two weeks apart. Reward and punishment processing

9 parameters from the reinforcement learning model showed fair-to-good reliability, while

10 risk/loss aversion parameters from a prospect theory model exhibited good-to-excellent

11 reliability. Both models were further able to predict future behaviour above chance within

12 individuals. This prediction was better when based on participants' own model parameters than

13 other participants' parameter estimates. These results suggest that reinforcement learning, and

14 particularly prospect theory parameters, can be measured reliably to assess learning and

15 decision-making mechanisms, and that these processes may represent relatively distinct

16 computational profiles across individuals. Overall, these findings indicate the translational

17 potential of clinically-relevant computational parameters for precision psychiatry.

19 Keywords: Computational psychiatry, Reliability, Decision-making, Reinforcement learning,

20 Prospect Theory, Gambling 


\section{Introduction}

2 Cognitive and neural processes are increasingly conceptualized in computational terms

3 (Palminteri, Wyart, \& Koechlin, 2017). Generative computational models offer the advantage of

4 examining behaviourally unobservable, but important, latent processes that drive behaviour

5 and can be closely linked to neurobiology (Huys, Maia, \& Frank, 2016; Montague, Hyman, \&

6 Cohen, 2004). As such, they provide a mathematically precise framework for specifying

7 hypotheses about the cognitive processes that generate behaviour. These features make

8 modelling a powerful tool to provide mechanistic accounts into (a)typical behaviours, including

9 those associated with psychiatric symptoms.

11 The increasing adoption of computational approaches in cognitive neuroscience inspired the

12 emerging discipline of computational psychiatry, which aims to better understand mental

13 illness through computational methods with the ultimate goal of transforming such knowledge

14 into new personalised treatment strategies (Adams, Huys, \& Roiser, 2016; Browning et al.,

15 2020; Friston, Redish, \& Gordon, 2017; Huys, 2018; Huys et al., 2016; Huys, Moutoussis, \&

16 Williams, 2011; Maia \& Frank, 2011; Montague, Dolan, Friston, \& Dayan, 2012; Patzelt, Hartley,

17 \& Gershman, 2018; Paulus, Huys, \& Maia, 2016; Paulus \& Thompson, 2019; Teufel \& Fletcher,

18 2016; Wang \& Krystal, 2014; Wiecki, Poland, \& Frank, 2015). For such translational endeavours

19 to be successful, however, it is vital that computational measures capture individual

20 characteristics reliably (Browning et al., 2020; Paulus et al., 2016). 
1 One area of computational modelling that has received particular attention is the reward and

2 punishment processes underlying decision-making (Maia, 2009). This progress has fostered

3 research into the computational mechanisms that may drive psychiatric symptoms, as

4 disrupted decision-making and reward processing are important features of many disorders

5 (Aylward et al., 2019; Browning, Behrens, Jocham, O'Reilly, \& Bishop, 2015; Diagnostic and

6 statistical manual of mental disorders: DSM-5, 2013; Eshel \& Roiser, 2010; Gillan, Kosinski,

7 Whelan, Phelps, \& Daw, 2016; Huys, Browning, Paulus, \& Frank, 2021; Huys et al., 2016;

8 Mkrtchian, Aylward, Dayan, Roiser, \& Robinson, 2017; Mkrtchian, Roiser, \& Robinson, 2017;

9 Robinson \& Chase, 2017). In particular, two classes of computational models, respectively

10 originating from computer science and behavioural economics, have been influential in

11 characterizing the cognitive mechanisms underlying decision-making: reinforcement learning

12 (RL) and prospect theory (Dayan \& Niv, 2008; Kahneman \& Tversky, 1979; Maia, 2009; Niv,

13 2009; Schonberg, Fox, \& Poldrack, 2011; Sutton \& Barto, 2018; Tversky \& Kahneman, 1992).

15 RL models describe how agents learn from rewards and punishments through trial-and-error

16 (Sutton \& Barto, 2018). Within the field of computational psychiatry, this set of models has

17 perhaps been the most influential. For example, reward and punishment sensitivity (reflecting

18 subjective valuation of the outcomes) and learning rate (reflecting how quickly individuals learn

19 from better- or worse-than-expected outcomes) have been associated with distinct

20 symptomatology and neural signals (Daw \& Doya, 2006; Huys et al., 2021; Maia \& Frank, 2011;

21 Niv, 2009). A commonly-used RL task is the multi-armed "bandit", in which individuals choose

22 between multiple slot machines with fluctuating, unknown probabilities of reward and 
1 punishment, with the goal of maximizing earnings (Cohen, McClure, \& Yu, 2007; Daw,

2 O'Doherty, Dayan, Seymour, \& Dolan, 2006; Seymour, Daw, Roiser, Dayan, \& Dolan, 2012;

3 Speekenbrink \& Konstantinidis, 2015; Yi, Steyvers, \& Lee, 2009). Participants must decide on

4 each trial whether to persist with the previously sampled slot machine or explore others which

5 may yield better outcomes. The mechanisms thought to underlie these decisions can be

6 captured effectively by RL models (Aylward et al., 2019; Daw et al., 2006).

7

8 Prospect theory models, on the other hand, describe the cognitive processes driving decision-

9 making biases under known risks, and have been extremely influential in understanding

10 economic decision-making (Kahneman \& Tversky, 1979; Ruggeri et al., 2020; Schonberg et al.,

11 2011; Sokol-Hessner \& Rutledge, 2019; Tversky \& Kahneman, 1992). These processes are often

12 examined by asking participants to choose between a guaranteed outcome (e.g., $f 0$ gain) and a

$1350 \%$ gamble with two possible outcomes (e.g., $£ 30$ gain or $£ 10$ loss). It is commonly observed

14 that humans tend to prefer a sure payment over a risky payment with equivalent or higher

15 expected value. For example, you may prefer an investment with a fixed return over one with a

16 potentially higher but uncertain return. Prospect theory proposes that these observations can

17 be accounted for by two different processes: 1) risk aversion - the preference for certain over

18 uncertain gains, and 2) loss aversion - weighting losses more heavily than gains. Risk and loss

19 aversion vary across individuals, and these differences have been associated with psychiatric

20 diagnoses and affective states (Baek et al., 2017; J. K. Brown et al., 2013; Charpentier, Aylward,

21 Roiser, \& Robinson, 2017; Charpentier, De Martino, Sim, Sharot, \& Roiser, 2016; Charpentier,

22 De Neve, Li, Roiser, \& Sharot, 2016; Hadlaczky et al., 2018; Hartley \& Phelps, 2012; Klaus, 
1 Chumbley, Seifritz, Kaiser, \& Hartmann-Riemer, 2020; Sip, Gonzalez, Taylor, \& Stern, 2017;

2 Tremeau et al., 2008). Importantly, computational modelling has allowed researchers to

3 dissociate risk and loss aversion and their contribution to symptoms (Charpentier et al., 2017).

4

5 Parameters from RL and prospect theory models show promise in generating insights into the mechanisms underlying psychiatric symptoms. However, as of yet, the psychometric properties

7 of computational parameters have received limited attention (Ahn \& Busemeyer, 2016;

8 Browning et al., 2020; Nair, Rutledge, \& Mason, 2020; Paulus et al., 2016). Model-agnostic measures derived from reward processing tasks (e.g., percent correct) often have modest or

10 poor reliability (Bland et al., 2016; Enkavi et al., 2019; Plichta et al., 2012), and the handful of

11 studies that have examined the reliability of computational parameters have reported markedly

12 varied results. For example, risk aversion parameters have been reported to be fairly stable in a

13 decision-making task in both healthy and depressed individuals (Chung et al., 2017). Similarly,

14 parameters from a prospect theory model based on a gambling task showed significant

15 correlations over time (Glockner \& Pachur, 2012; Scheibehenne \& Pachur, 2015). In contrast,

16 reliability assessment of parameters derived from a go/no-go RL task exhibited poor reliability

17 (Moutoussis et al., 2018), as did those from a two-step decision-making task assessing model-

18 based versus model-free RL (Shahar et al., 2019). Interestingly, the reliability of parameters

19 derived from the latter task was substantially improved through hierarchical estimation

20 procedures, suggesting that the estimation procedure matters (V. M. Brown, Chen, Gillan, \&

21 Price, 2020). However, only one of the above studies (V. M. Brown et al., 2020) quantified test-

22 retest reliability using the standard intraclass correlation coefficient (ICC), which allows 
1 assessing both the relative rank and absolute agreement between measurements (Koo \& Li,

2 2016). Additionally, no studies to date have reported the reliability of decision-making

3 processes derived from a bandit task with fluctuating ("restless") reward and punishment

4 probabilities (Daw et al., 2006) or an individually calibrated gambling task (Charpentier et al., 5 2017).

7 A complementary perspective to understanding the reliability of computational cognitive

8 models can be obtained through prediction. Generative models offer a substantial advantage in

9 that they can both explain and predict behaviour. Unlike model-agnostic measures,

10 computational parameters fit to one dataset should be able to predict future behaviour in the

11 same individual. In other words, computational models can additionally be assessed by their

12 ability to forecast future behaviour, equivalent to out-of-sample validation. This type of

13 validation assesses model generalizability and is often referred to as predictive accuracy

14 (Busemeyer \& Wang, 2000; Glockner \& Pachur, 2012; Scheibehenne \& Pachur, 2015), but it has

15 rarely been used as a metric of reliability. Therefore, in the current study we assessed the

16 reliability of computational parameters derived from two widely-used decision-making tasks (a

17 restless four-armed bandit and a calibrated gambling task) using both approaches: standard

18 measures of stability and reliability (respectively, practice effects and ICCs); and out-of-sample

19 predictive accuracy. 
1 Methods and Materials

2 Participants

3 Fifty-four healthy participants were recruited from the UCL Institute of Cognitive Neuroscience

4 Subject Database. Four participants were excluded for failing to complete the second session

5 (final $\mathrm{N}=50$ : 32 females [64\%]; age range=19-38; mean age=25.16, $\mathrm{SD} \pm 5.48$ years; mean

6 education $=17.38, \mathrm{SD}= \pm 3.24$ years). Participants reported no current or past psychiatric or

7 neurological disorder, cannabis use in the past 31 days, alcohol consumption in the past 24

8 hours, or any other recreational drug use in the week prior to participation. Participants

9 provided written informed consent and were compensated at the end of their second session

10 with a flat rate of $£ 30$ and a bonus of up to $£ 20$ based on task winnings. The study was

11 approved by the UCL Psychology and Language Sciences Research Ethics Committee (Project ID

12 Number: $\mathrm{fMRI} / 2013 / 005)$ and was performed in accordance with the Declaration of Helsinki.

14 Sample size was determined by an a priori power analysis in G*Power (Faul, Erdfelder, Lang, \&

15 Buchner, 2007). The power analysis was based on the smallest effect size of interest, $r=0.4$,

16 since reliability below this threshold is conventionally considered poor (Fleiss, 2011). Detecting

17 an effect size of this magnitude, at the one-tailed 0.05 alpha level with $90 \%$ power, requires 47

18 participants. 
1 Study procedure and tasks

2 Participants completed a battery of computerized tasks, including a restless four-armed bandit

3 (Daw et al., 2006; Seymour et al., 2012) and an individually calibrated gambling task

4 (Charpentier et al., 2017) over two sessions (mean test-retest interval $=13.96$ days, $S D=0.20$ ).

5 On each trial in the bandit task, participants chose one out of four bandits and received one out

6 of four possible outcomes: reward, punishment, neither reward nor punishment or both

7 reward and punishment. Win and loss probabilities fluctuated independently over time and

8 between boxes (Figure 1a; Supplemental Materials). In the gambling task, participants chose

9 between a 50-50 gamble and a sure option without receiving feedback. An initial training phase

10 was used to create individually calibrated offers (centred on indifference points) in a second

11 phase. Trials were classified as either mixed ( $50 \%$ chance to win or lose money gamble, or a

12 sure option of 0 points) or gain-only ( $50 \%$ chance to win or receive nothing gamble, or a

13 variable sure gain; Figure 1b; Supplemental Materials). Calibration failed for one participant

14 resulting in $\mathrm{N}=49$ participants for this task. 
a

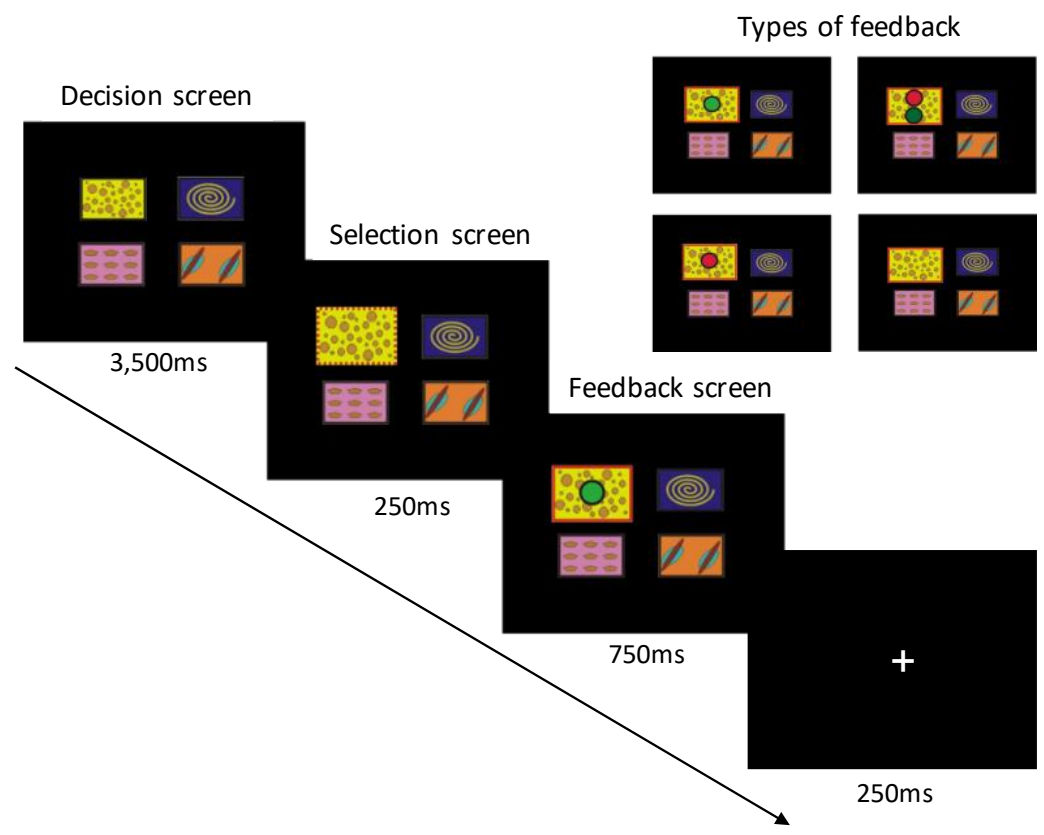

b

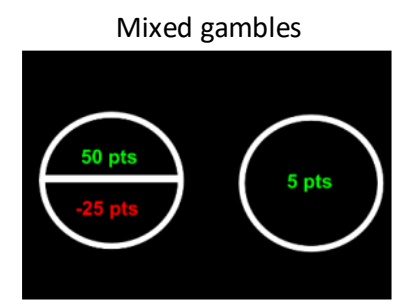

Gain-only gambles

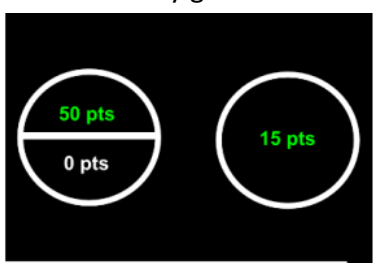

3 Figure 1. Four-armed bandit and gambling task. Example trial of the four-armed bandit task (a). On each trial, participants chose one out of four bandits and received one out of four possible outcomes: reward (green token),

5 punishment (red token), neither reward nor punishment (empty box) or both reward and punishment (red and

6 green token). On each gambling task trial, participants chose between a 50-50 gamble and a sure (guaranteed

7 amount of points) option (b). Trials were either mixed gambles (50-50 chance of winning or losing points or sure

8 option of 0 points) or gain-only trials (50-50 chance of winning or receiving nothing or sure gain).

9

Data analysis

11 Data were processed in Matlab (R2019b) and analysed in SPSS (v25, IBM Corp, Armonk, NY).

12 Computational modelling was performed with the hBayesDM package for R (v. 3.6.0;

13 https://github.com/CCS-Lab/hBayesDM; Ahn, Haines, \& Zhang, 2017), which uses hierarchical

14 Bayesian modelling in Stan (v.2.21.2). For all analyses, $p<0.05$ (two-tailed) was considered 
1 statistically significant. Cohen's $d_{z}$ (within-subject) effect sizes are reported for practice effects

2 (Lakens, 2013). Details on model-agnostic task measures are in Supplemental Materials.

5 The bandit task data was fit with seven different RL models previously described in detail

6 (Aylward et al., 2019; Supplemental Materials). Three prospect theory models were fit to the

7 gambling task (Ahn et al., 2017; Charpentier et al., 2017; Kahneman \& Tversky, 1979; Sokol-

8 Hessner et al., 2009). Modelling was conducted on the second phase of the gambling task (i.e.,

9 on individually calibrated trials). The models were fit for each session separately, using separate

10 hierarchical priors (group-level parameters), as this has shown to provide more accurate fits

11 (Valton, Wise, \& Robinson, 2020), and we wished to avoid artificially inflating reliability

12 estimates. We also estimated model fits under a single hierarchical prior (session 1 and session

132 data together) as a sensitivity analysis (Supplemental Materials). Model comparison was

14 performed with leave-one-out information criterion (LOOIC) where the winning model was the

15 one with the lowest LOOIC. Several model validation checks were completed for the winning

16 models (Daw, 2011; Kruschke, 2015; Wilson \& Collins, 2019; Supplemental Materials).

18 Reliability analysis

19 Test-retest reliability was assessed with ICCs (ratios of intra-individual to inter-individual

20 variability (Koo \& Li, 2016; McGraw \& Wong, 1996), with values of <0.40 interpreted as poor,

$21 \quad 0.4-0.6$ as fair, 0.6-0.75 as good, and $>0.75$ as excellent reliability (Fleiss, 2011). A two-way

22 mixed effects model based on single measures ICC was used (fixed effect: testing time-interval, 
1 random effect: subject). We report consistency ICCs here (assesses relative ranking of

2 participants over time) but note that there is little difference compared with absolute ICCs

3 (additionally assess changes in scores over time; Supplemental Materials).

\section{$5 \quad$ Posterior predictive performance}

6 To assess to what extent an individual's future behaviour can be predicted using a generative

7 model fit to their own task performance two weeks earlier, we calculated the probability of

8 participants' choices on each trial (i.e., the softmax output), given their session 2 data and

9 model parameter estimates from session 1. Probabilities were averaged across trials for each

10 individual.

12 Since hierarchical parameter estimation produces 'shrinkage', effectively pulling parameter

13 estimates from different individuals closer to each other (which improves estimation accuracy),

14 it is possible that future performance may also be predicted above-chance using other

15 participants' parameter estimates from session 1 (e.g., participant A's parameter estimates

16 from session 1 predicting participant B's session 2 choices). We therefore assessed whether

17 using an individual's model parameter estimates from session 1 predicted the same individual's

18 choices on session 2 better than using all other subjects' model parameter estimates. To

19 construct the latter measure, for each subject, we predicted trial-by-trial choices on session 2

20 based on parameter estimates from every other participant's session 1 model, and averaged

21 the probabilities across all participants. 


\section{Results}

2 Four-armed bandit task: model-agnostic results

3 Basic behaviour and practice effects

4 As expected, there was a main effect of outcome type on behaviour $\left(F_{(2,98)}=117.39, p<0.001\right.$,

$5 \quad \eta_{p}^{2}=0.71 ;$ Figure $\left.2 a\right)$. The probability to repeat a choice was significantly greater after wins

6 compared with both losses and outcomes on which neither wins nor losses occurred, and

7 greater after neither compared with losses (all $p<0.001$ ). There was no significant main effect of

8 testing session $\left(F_{(1,49)}=0.01, p=0.91, \eta_{p}^{2}<0.001\right)$, but there was a significant outcome-by-session

9 interaction $\left(F_{(2,98)}=3.12, p=0.049, \eta_{p}^{2}=0.06\right)$, reflecting slightly increased repeated choices after

10 wins and decreased repeated choices after losses. However, the difference in the tendency to

11 repeat a choice between session 1 and session 2 did not reach significance following any of the

12 outcome types (loss: $\mathrm{t}_{(49)}=1.45, p=0.15, \mathrm{~d}_{\mathrm{z}}=0.21$; win: $\mathrm{t}_{(49)}=0.87, p=0.39, \mathrm{~d}_{\mathrm{z}}=0.12$; neither:

$\left.13 t_{(49)}=0.54, p=0.59, d_{z}=0.08\right)$, and therefore we do not interpret this result further.

14

15 Test-retest reliability

16 The model-agnostic outcome measures of the bandit task exhibited good reliability (Figure $2 \mathrm{~b}$ ). 
a

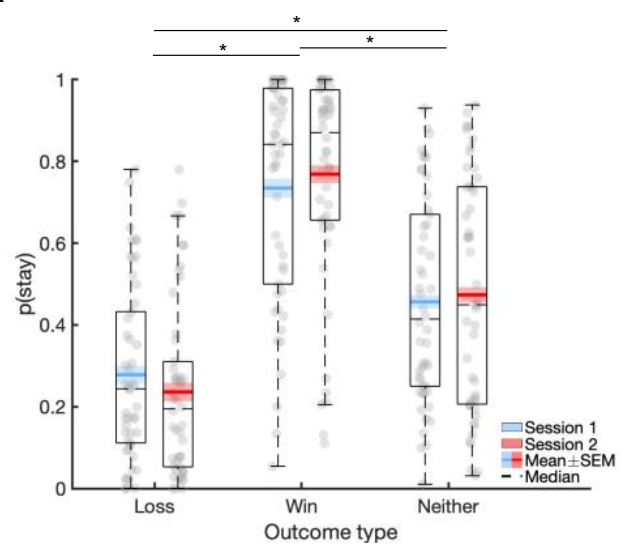

b

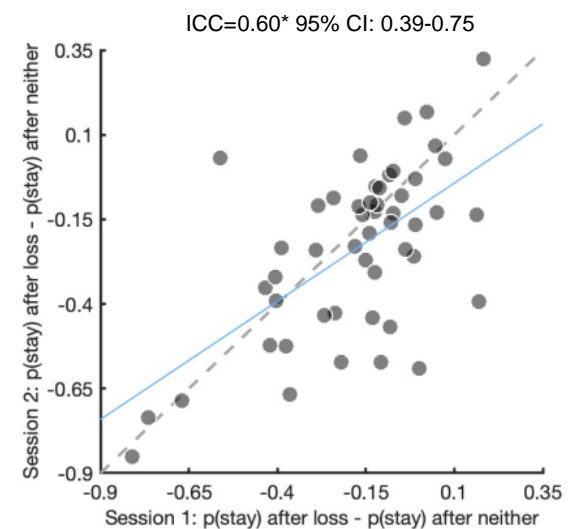

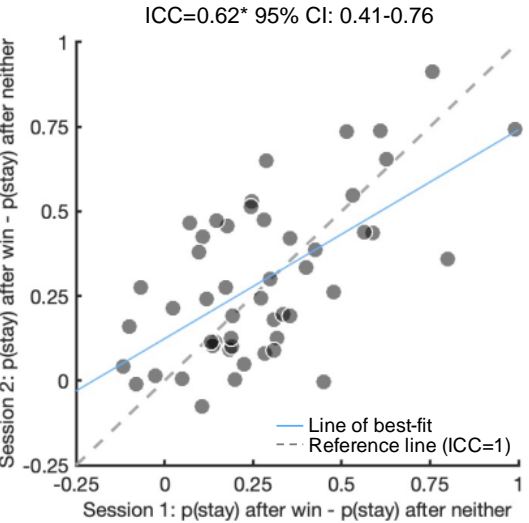

1 Figure 2: Basic behaviour, practice effects, and test-retest reliability of model-agnostic measures on the four-

2 armed bandit task. Boxplots of the four-armed bandit task showing probability to stay after a certain outcome in

3 session 1 and 2 (a). The probability to stay was significantly different after each outcome type (Loss $<$ Neither $<$ Win)

4 but no clear practice effect was evident. Scatter plots and reliability of the four-armed bandit task model-agnostic

5 measures comparing behaviour on two testing sessions approximately 2 weeks apart (b). Consistency (assesses

6 relative ranking over time) intraclass correlation coefficients (ICC) are presented. Lightly shaded regions in Figure

7 2a represent within-subjects standard error of the mean (SEM). Cl: confidence interval. ${ }^{*} p<0.001$

$9 \quad$ Four-armed bandit task: modelling results

10 Model comparison indicated that the winning (most parsimonious) model was the five-

11 parameter 'Bandit4arm_lapse' (nomenclature from the hBayesDM package) model, with

12 reward and punishment learning rate parameters, reward and punishment sensitivity

13 parameters and a lapse parameter (final parameter captures random responding; Table S1),

14 consistent with previous reports (Aylward et al., 2019). Several model diagnostics were

15 conducted to check the model performance (Figure S1-5). Three individuals were excluded due

16 to difficulties in obtaining mean parameter estimates, as multiple peaks were evident in the

17 posterior distribution of at least one parameter (Figure S2). The Bandit4arm_lapse model was 
1 therefore re-fit without these participants. Excluding these participants did not affect test-

2 retest reliability inference (Table S2). All parameters other than the lapse parameter showed

3 high recoverability (Figure S3), and synthetic data from the winning model accurately

4 recapitulated real data (Figure S4).

$6 \quad$ Practice effects

7 On session 2 there were significant increases in the reward sensitivity $\left(t_{(46)}=3.00, p=0.004\right.$,

$\left.8 d_{z}=0.44\right)$ and lapse parameters $\left(t_{(46)}=8.88, p<0.001, d_{z}=1.29\right)$, but not on any of the other

9 parameters (reward learning rate: $\mathrm{t}_{(46)}=1.28, p=0.21, \mathrm{~d}_{\mathrm{z}}=0.19$; punishment learning rate:

$10 \mathrm{t}_{(46)}=1.74, p=0.09, \mathrm{~d}_{\mathrm{z}}=0.25$; punishment sensitivity: $\mathrm{t}_{(46)}=1.28, p=0.21, \mathrm{~d}_{\mathrm{z}}=0.19 ;$ Figure $3 a$ ).

11 However, there were no significant practice effects when the data was fit under a single

12 hierarchical prior (Table S3).

14 Test-retest reliability

15 All estimated Bandit4arm_lapse model parameters, except the lapse parameter, demonstrated

16 fair-to-good reliability (Figure 3b), which did not substantially change when parameters were

17 estimated under a single hierarchical prior (Table S3). 
a
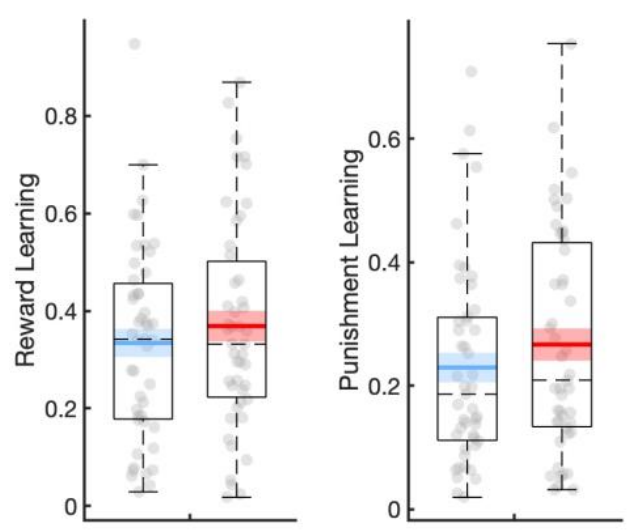
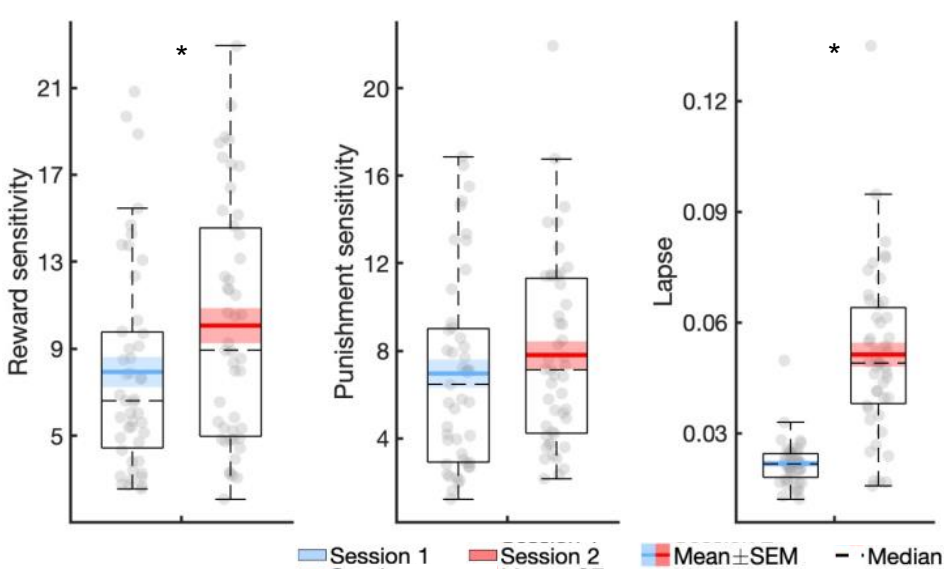

b
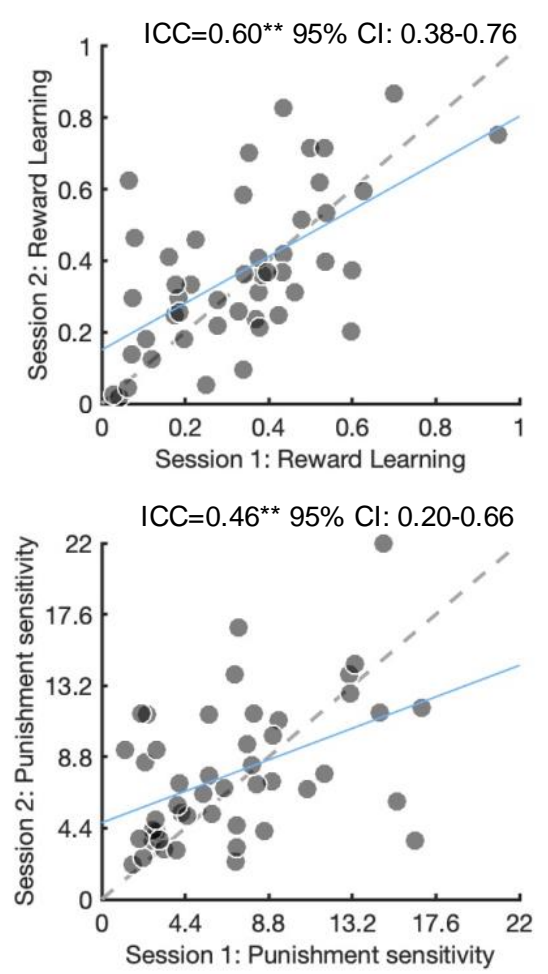
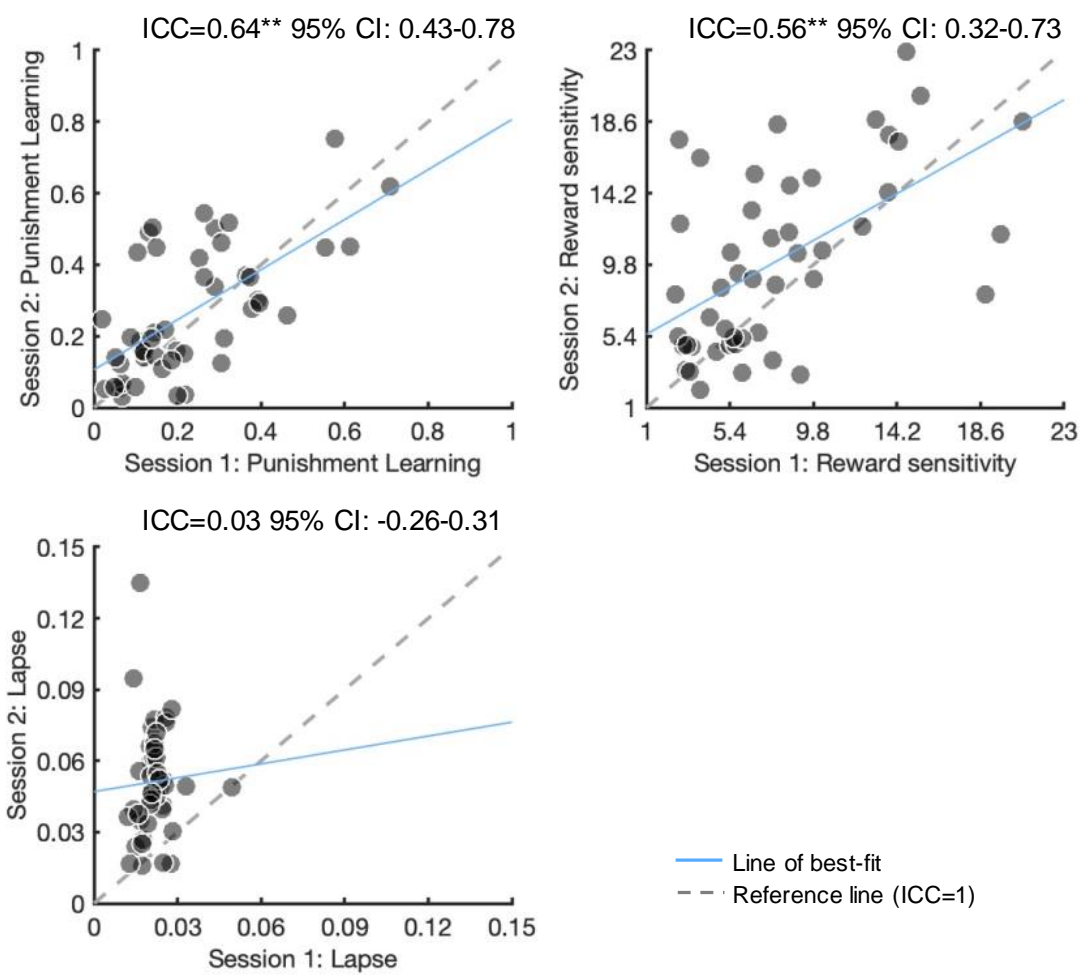

- Line of best-fit - - Reference line $(I C C=1)$

\section{Figure 3: Practice effects and test-retest reliability of the winning reinforcement learning model parameters}

3 derived from the four-armed bandit task. Boxplots show point estimates of the Bandit4arm_lapse model

4 parameters in session 1 and 2, fit under separate priors (a). Scatter plots and reliability of the Bandit4arm_lapse

5 model parameters over session 1 and 2 are presented (b). Consistency (assesses relative ranking score over time)

6 intraclass correlation coefficients (ICC) are presented. SEM: standard error of the mean; Cl: confidence interval; S1:

7 session 1 ; S2: session $2 .{ }^{*} p<0.05,{ }^{* *} p<0.001$ 


\section{Posterior predictive performance}

2 Parameter estimates from session 1 predicted task performance on session 2 substantially

3 better than chance ( mean $=42 \%$, chance $=25 \%$ accuracy; $t_{(46)}=9.10, p<0.001$; Figure $\left.4 a\right)$,

4 indicating that the model could predict future choices by using a generative model fit to the

5 same participants' data two weeks earlier. Using an individual's parameter estimates to predict

6 their own future choices was significantly better than when that prediction was based on the

7 average of the other participants' session 1 estimates $\left(t_{(46)}=3.20, p=0.003\right.$; Figure $\left.4 b\right)$, signifying

8 good individual-level model generalizability.

a

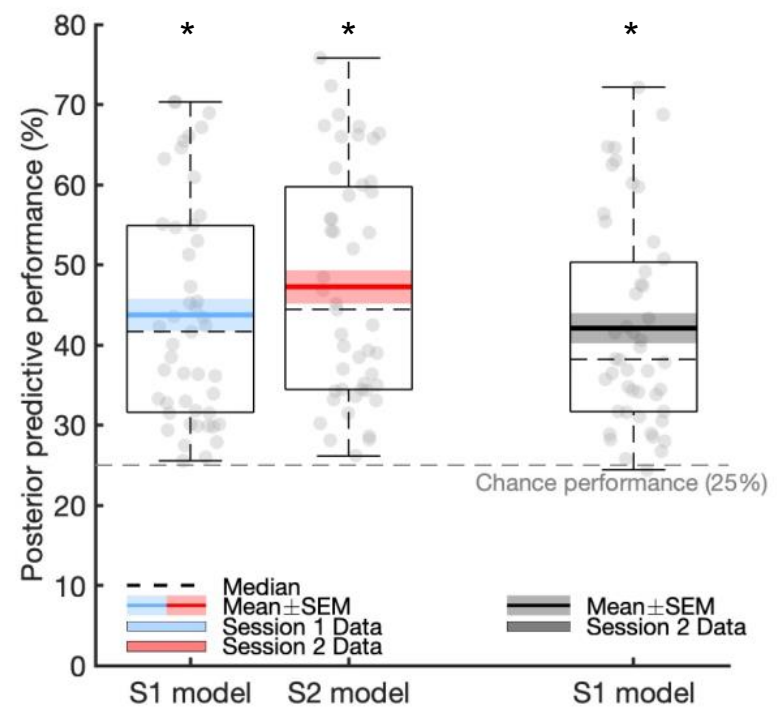

b

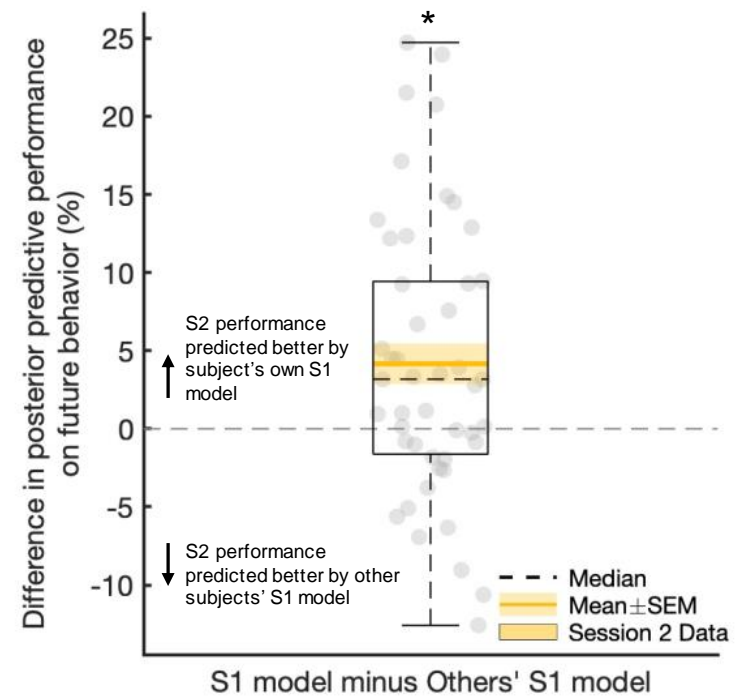

11

12 Figure 4: Posterior predictive performance of the winning reinforcement learning model derived from the four-

13 armed bandit task. Boxplots depicting accuracy of bandit4arm_lapse model in predicting choices (a). Model

14 estimates from session 1 (S1) predicted future session 2 (S2) behaviour above chance (black boxplot). Both S1 and

15 S2 model estimates also predicted behaviour on the same session significantly above chance (blue and red 
1 boxplots). Predicting future performance (session 2 data) using a participant's own model parameter estimates

2 was significantly better than using other participants' S1 model parameter estimates (b - zero indicates mean

3 accuracy from other participants' parameter estimates). SEM: standard error of the mean. ${ }^{*} p<0.01$.

4

$5 \quad$ Gambling task: model-agnostic results

6 Basic behaviour and practice effects

7 As expected, propensity to gamble was significantly higher on mixed gambles $\left(F_{(1,48)}=13.71\right.$,

$\left.8 p=0.001, \eta_{p}^{2}=0.22\right)$. There were no significant main $\left(F_{(1,48)}=0.76, p=0.40, \eta_{p}^{2}=0.02\right)$ or interaction

$9 \quad\left(F_{(1,48)}=1.07, p=0.31, \eta_{p}^{2}=0.02\right)$ effects of session on the propensity to gamble (session

10 differences: probability to gamble on mixed trials $t_{(48)}=0.23, p=0.82, d_{z}=0.03$; probability to

11 gamble on gain-only trials $\mathrm{t}_{(48)}=1.51, p=0.14, \mathrm{~d}_{2}=0.22$; Figure $5 \mathrm{a}$ ).

12

13 Test-retest reliability

14 Model-agnostic outcome measures on the gambling task exhibited good reliability (Figure 5b).

15

16

17

18

19

20

21 
a

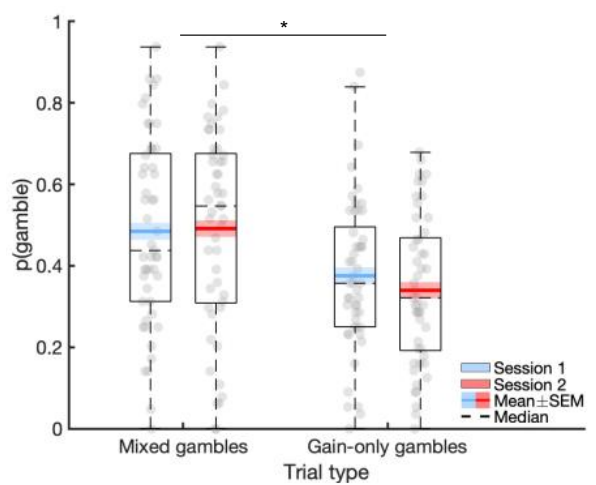

b

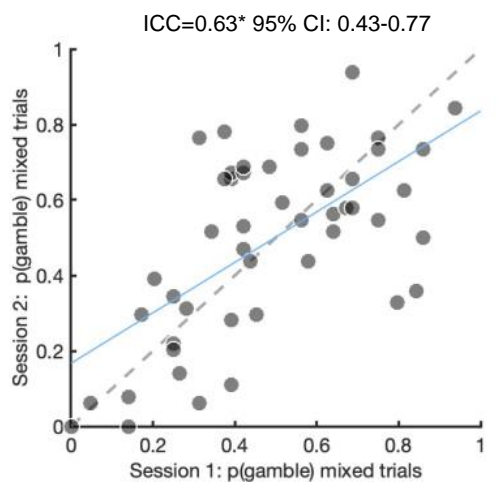

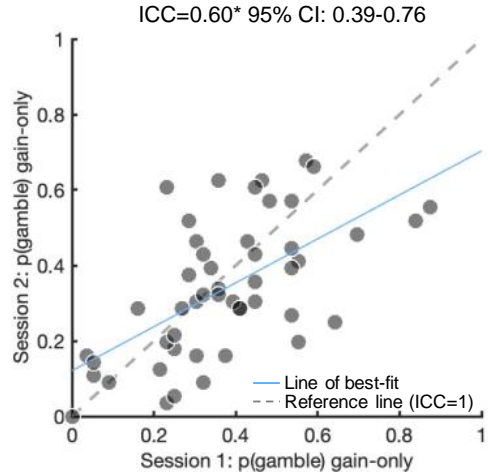

1 Figure 5: Basic behaviour, practice effects, and test-retest reliability of model-agnostic measures on the

2 gambling task. Boxplots show the probability to gamble based on the trial type in session 1 and 2 , with no

3 significant session effects (a). Scatter plots and reliability of the gambling task model-agnostic measures over

4 session 1 and 2 (b). Consistency (assesses relative score ranking over time) intraclass correlation coefficients (ICC)

5 are presented. Lightly shaded regions in Figure 5a represent within-subjects standard error of the mean (SEM). Cl:

6 confidence interval. ${ }^{*} p<0.001$.

8 Gambling task: modelling results

9 The winning model was the prospect theory model ('ra_prospect' in the hBayesDM package)

10 with loss aversion, risk aversion and inverse temperature parameters (last parameter

11 represents choice consistency; Table S4), consistent with previous reports (Charpentier et al.,

12 2017). A loss aversion parameter above 1 represents overweighting of losses to gains, while a

13 risk aversion parameter less than 1 indicates aversion to risk. Model diagnostics indicated that

14 this model performed well (Figure S6). All parameters showed high recoverability (Figure S7),

15 and synthetic data from the winning model accurately recapitulated real data (Figure S8).

16 Neither test-retest nor practice effects were substantially altered when the model was fit under

17 a single hierarchical prior (Table S5). 


\section{Practice effects}

3 There were significant session effects on all prospect theory model parameters (on session 2:

4 decreased loss aversion: $\mathrm{t}_{(48)}=2.17, p=0.04, \mathrm{~d}_{\mathrm{z}}=0.31$; decreased risk aversion: $\mathrm{t}_{(48)}=4.04, p<0.001$,

$5 \quad d_{z}=0.58$; increased inverse temperature: $t_{(48)}=3.07, p=0.004, d_{z}=0.44$; Figure 6a).

8 All estimated parameters demonstrated good-to-excellent reliability (Figure 6b).

a
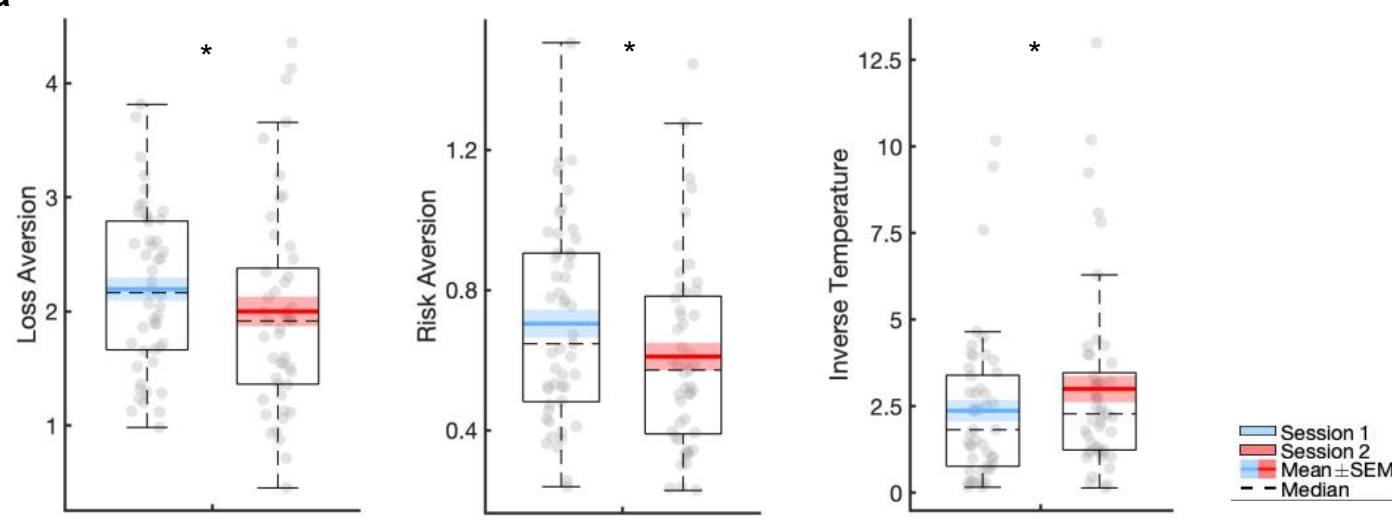

b
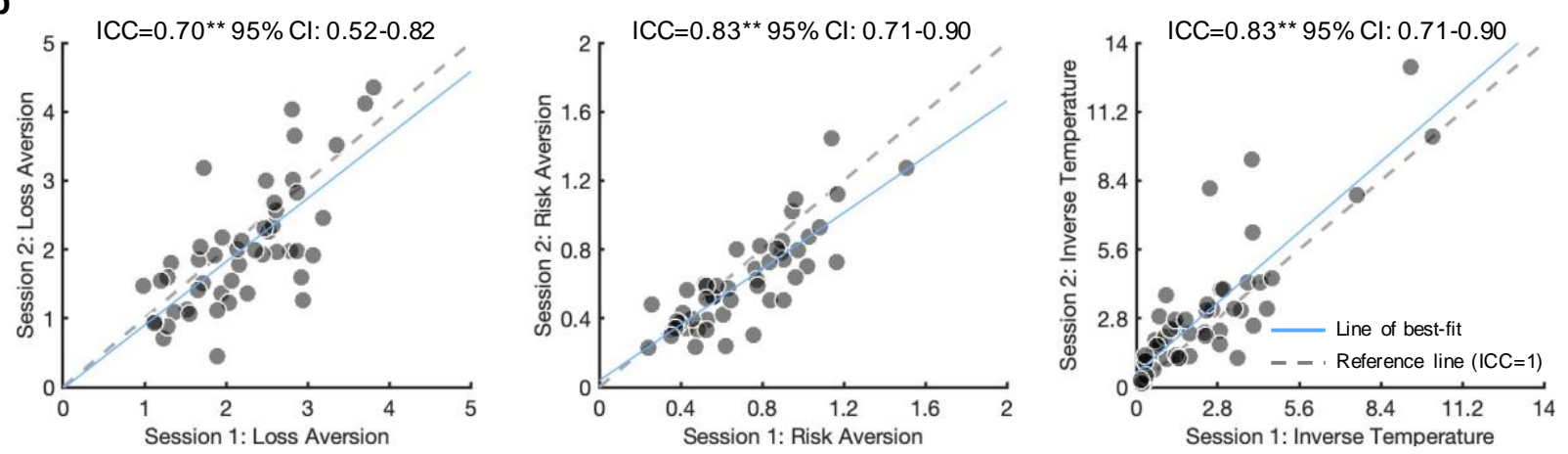

10

11 Figure 6: Practice effects and test-retest reliability of the prospect theory model derived from the gambling task.

12 Boxplots show point estimates of the prospect theory model parameters in session 1 and 2, fit under separate 
1 priors (a). Scatter plots and reliability of the prospect theory model parameters over session 1 and 2 are presented

2 (b). Consistency (assesses relative score ranking over time) intraclass correlation coefficients (ICC) are presented.

3 SEM: standard error of the mean; Cl: confidence interval; S1: session 1; S2: session 2. ${ }^{*} p<0.05,{ }^{* *} p<0.001$.

6 Prospect theory model parameters from session 1 predicted future choices at session 2

7 significantly above chance $\left(\right.$ mean $=68 \%$, chance $=50 \%$ accuracy; $\mathrm{t}_{(48)}=12.08, p<0.001$; Figure

8 7a). Predicting future performance at session 2 was significantly higher when based on

9 participants' own parameter estimates from session 1 compared with model parameter

10 estimates of other participants from session $1\left(t_{(48)}=8.38, p<0.001\right.$; Figure $\left.7 b\right)$.

11

a

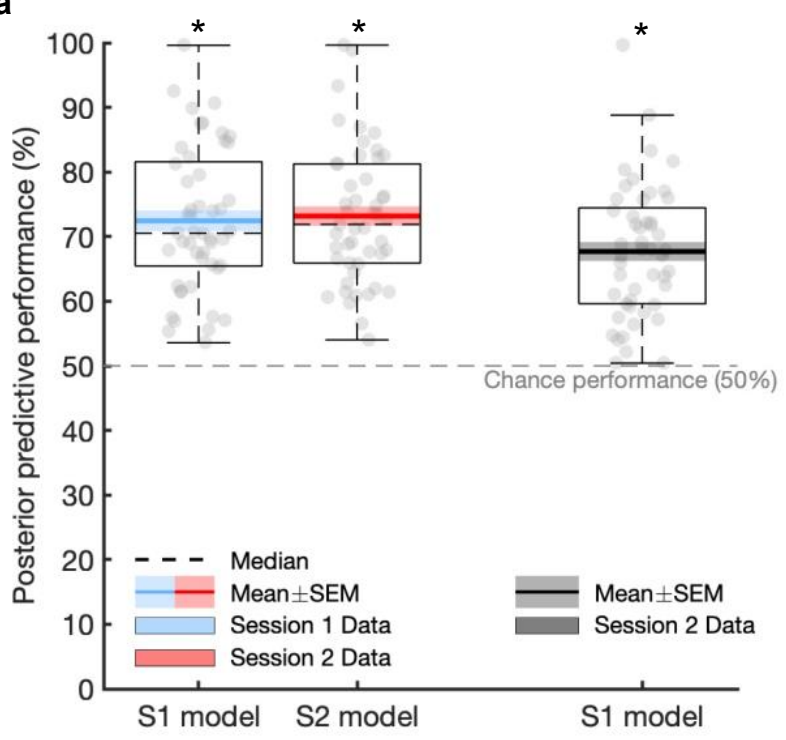

b

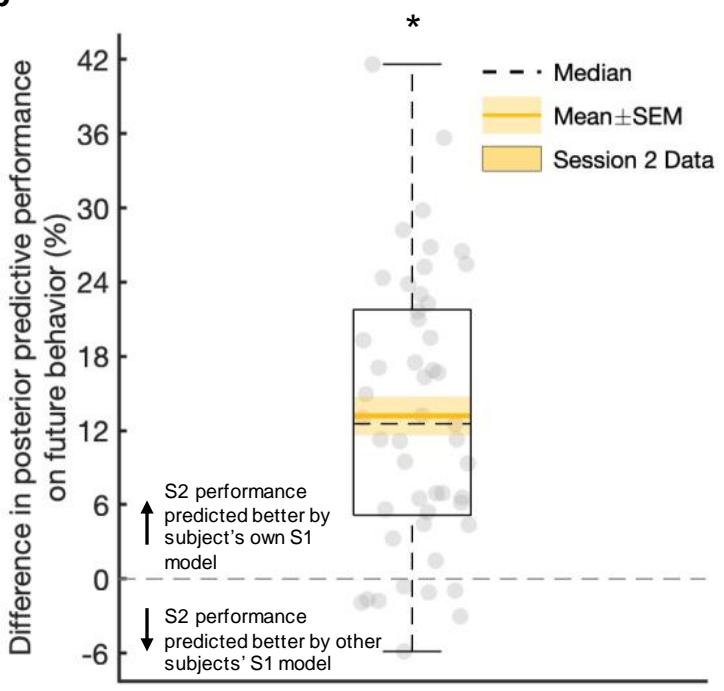

S1 model minus Others' S1 model

13 Figure 7: Posterior predictive performance of the prospect theory model derived from the gambling task.

14 Boxplots depicting accuracy of prospect theory model in predicting choices (a). Session 1 (S1) model estimates

15 predicted S1 behaviour significantly above chance (blue boxplot), as did session 2 (S2) model estimates on S2 data

16 (red boxplot). Importantly, model parameter estimates from S1 predicted task performance from S2 above chance 
1 (black boxplot). Predicting future S2 performance using a participant's own S1 model parameter estimates was

2 significantly better than using other participants' S1 model parameter estimates (b - zero indicates mean accuracy

3 from other participants' parameter estimates). SEM: standard error of the mean. * $p<0.001$.

7 across conventional measures from cognitive tasks and functional neuroimaging (Elliott et al.,

8 2020; Enkavi et al., 2019; Frey, Pedroni, Mata, Rieskamp, \& Hertwig, 2017; Noble, Scheinost, \&

9 Constable, 2019; Nord, Gray, Charpentier, Robinson, \& Roiser, 2017; Rodebaugh et al., 2016).

10 However, few attempts have been made to examine the reliability of computational cognitive

11 measures. Here we assessed the psychometric properties of computational models derived

12 from a restless four-armed bandit and a calibrated gambling task. Overall, most parameters

13 reflecting $\mathrm{RL}$ and decision-making processes exhibited adequate reliability and predicted future

14 performance well. These results provide promise for their use in clinical settings. However, this

15 conclusion depends on the specific parameters assessed in each task, highlighting the complexities of translating tasks to the clinic.

20 more suitable for assessing learning rates than sensitivity. Elevated punishment learning rates

21 (faster learning in the face of negative outcomes) and lapse values (random responding or

22 exploration) have been associated with greater mood and anxiety symptoms, representing 
1 potential measurable mechanistic treatment targets (Aylward et al., 2019). However, the

2 present study suggests that the lapse parameter, which exhibited poor reliability as assessed by

3 the bandit task, may not be suitable as a potential target. This parameter measures responding

4 not captured by the model (including goal-directed and random exploration), and the sources

5 of this 'noise' might differ across sessions. It is therefore perhaps unsurprising that this

6 parameter was unreliable. Crucially, the lapse parameter showed poor recoverability, which

7 places an upper limit on its potential reliability. Some of this poor recoverability may be

8 explained by limited lapse variation, especially in session 1 (Figure S3). The distribution of the

9 group-level standard deviation lapse parameter was biased towards smaller values here such

10 that the lapse parameter did not vary greatly between individuals (Figure S5). This suggests that

11 the lapse parameter could be replaced with a constant and inference on this parameter is not

12 advised.

14 Although no prior studies have specifically investigated ICC properties of the current RL model, 15 one previous study found similarly poor reliability of the lapse parameter across six months in a 16 go/no-go RL model in adolescents (Moutoussis et al., 2018). In contrast to our results, this study

17 also reported poor reliability of both reward and punishment learning rates. These differences

18 may arise for a multitude of reason. Importantly, however, we provide evidence that it is

19 possible to achieve at least moderate reliability for some canonical RL parameters.

21 Interestingly, the model-agnostic outcome measures of the bandit task exhibited similar

22 reliability to the computational measures. Model-agnostic measures of cognitive tasks have 
1 often been reported to exhibit poor-to-moderate reliability (Enkavi et al., 2019; Hedge, Powell,

2 \& Sumner, 2018; Plichta et al., 2012; Rodebaugh et al., 2016). It has been argued that this may

3 be due to their inability to capture the generative process underlying task performance (Huys et

4 al., 2021; Price, Brown, \& Siegle, 2019). Our results suggest that it should not be assumed that

5 computational parameters will always provide greater reliability than non-computational ones.

6 However, the model-agnostic outcome measures are only a proxy of the processes the bandit

7 task assesses, as it is difficult to compute model-agnostic equivalents of some parameters, such

8 as reward/punishment sensitivity. Indeed, models make explicit and falsifiable predictions of

9 the components driving behaviour, which can be refined and used to simulate artificial data to

10 generate new predictions. Thus, computational modelling is a more rigorous and preferable

11 method for assessing behaviour than model-agnostic measures, which unlike computational

12 methods, lack the mechanistic insights into the underlying processes generating behaviour.

14 While reliability of parameters ranged from poor-to-good in the bandit task, all parameters

15 from the gambling task showed good-to-excellent test-retest reliability. These were also

16 substantially higher than the reliability of the model-agnostic measures, suggesting that

17 computational models may offer advantages in psychometric properties here, in addition to

18 their advantage in specifying mechanisms. In particular, the risk aversion parameter, which has

19 previously been associated with anxiety (Charpentier et al., 2017), exhibited excellent reliability

20 (ICC>0.8), providing promise for use in clinical research. These results are consistent with

21 previous studies, but we generally found considerably higher reliability than previously

22 reported (previous reliability reports: loss aversion $r \approx 0.25-0.61$, risk aversion $r \approx 0.50-0.60$, 
1 inverse temperature $r \approx 0.30-0.60$ (Chung et al., 2017; Glockner \& Pachur, 2012; Scheibehenne \&

2 Pachur, 2015). These studies all used different estimation procedures, including hierarchical

3 Bayesian, and employed both longer and shorter testing time-windows than the current study,

4 suggesting that these factors may not fully explain the differences. It is possible that our results

$5 \quad$ instead stem from different prospect model specifications (Chung et al., 2017; Scheibehenne \&

6 Pachur, 2015), as well as different task designs.

7

8 The mean loss and risk aversion parameters observed are consistent with previous literature

9 indicating that losses are weighed about twice as much as gains, and that people are on

10 average risk averse (Kahneman \& Tversky, 1979; Schonberg et al., 2011; Sokol-Hessner \&

11 Rutledge, 2019; Tversky \& Kahneman, 1992). Indeed, prospect theory models have shown to be

12 highly replicable across different contexts (Ruggeri et al., 2020). This implementation of the

13 gambling task may therefore represent a case where group-average results are highly

14 reproducible but the task is also suitable to assess individual differences. It has been observed

15 that this is rarely the case, since tasks that are designed to produce reliable average effects do

16 so by minimizing between-subject variance, whereas what makes tasks reliable and suitable for

17 inter-individual assessments are large between-subject but small within-subject variances

18 (Hedge et al., 2018). This may have occurred because of the calibrated design of the present

19 gambling task. A similar approach of dynamically updating parameter values to each individual

20 during task performance has previously been suggested as a solution to unreliable cognitive

21 tasks (Palminteri \& Chevallier, 2018). 
1 In addition to examining reliability using the traditional ICC method, we also examined how well

2 the models predicted future task performance. This approach provides complementary

3 perspective on reliability, which is unique to computationally-informed measures. Generative

4 models were consistently able to predict participants' future behaviour above chance. Notably,

5 on average, each participant's own parameter estimates from the first session were better at

6 predicting their future performance compared with using parameter estimates from all other

7 participants. This indicates that individuals may also reliably differ in the cognitive mechanisms

8 underlying their decisions, and offers reassurance that hierarchical estimation procedures are

9 suitable for inter-individual inferences (V. M. Brown et al., 2020; Daw, 2011; Scheibehenne \&

10 Pachur, 2015). In other words, individuals show relatively unique computational decision-

11 making profiles. This is consistent with two previous studies using a different prospect theory

12 model and gambling task (Glockner \& Pachur, 2012; Scheibehenne \& Pachur, 2015).

14 Some of the reliable parameters, however, showed small-to-medium session effects. Practice

15 effects cans either obscure a true effect or lead to false treatment claims if appropriate controls

16 are not employed. Quantifying session effects, however, allows such changes to be accounted

17 for. In the RL model, reward sensitivity increased in the second session, while all other reliable

$18 \mathrm{RL}$ processes were fairly stable. In contrast, all prospect theory parameters showed significant

19 session effects. However, the sensitivity analysis showed no substantial practice effects of RL

20 parameters when data were fit under a single prior, while the practice effects of prospect

21 theory parameters remained. Thus, the apparent practice effects on the RL task should be

22 interpreted with caution, as these may be overestimated under the two prior estimation 
1 approach (Valton et al., 2020). Importantly, the ICC estimates were hardly influenced by the

2 estimation approach. Although practice effects were evident in the computational parameters,

3 no clear changes were observed in the model-agnostic measures, suggesting further that

4 model-agnostic measures lack the precision to quantify these decision-making processes.

6 A potential limitation of our study is the sample tested, as the reliability of tasks in healthy

7 individuals may differ from that in clinical groups. Similarly, our results only speak to reliability

8 over two weeks. Thus, it is possible that longer time periods may produce lower reliability,

9 which should be assessed in future studies.

11 The RL parameters showed relatively modest reliability, suggesting that these processes are

12 more vulnerable to state influences than prospect theory parameters. A consequence of this is

13 that larger sample sizes may be required to examine effects, as effect sizes would be expected

14 to be lower, relative to prospect theory tasks (Fleiss, 2011). Interpreting the marked difference

15 in reliability between the prospect theory and RL models is not straightforward, as these

16 models measure complementary aspects of cognition. The bandit task is a learning paradigm

17 that requires constant updating of optimal choices. It is possible that in the first session

18 individuals had not yet stabilized on a cognitive strategy and were still learning the task

19 structure, as indicated by lower evidence of the winning model in session 1 compared with

20 session 2 (Table S1). It would be interesting to explore if an initial baseline session, familiarizing

21 participants with the task, would improve test-retest reliability. It is also possible that 
1 estimating test-retest reliability within a generative model, may provide a more accurate

2 theoretical representation of reliability (V. M. Brown et al., 2020; Haines et al., 2020); for this

3 reason we may have slightly underestimated reliability.

4

5 In summary, we show that commonly-used computational parameters derived from an RL

6 'restless' bandit task and a calibrated gambling task exhibit fair-to-excellent reliability. These

7 models can further be used to predict future behaviour in the same individuals, indicating that

8 the RL and decision-making processes assessed in these tasks represent relatively consistent

9 and unique characteristics of an individual. These findings take us one step closer to translating

10 computational measures of behaviour into clinical application. 


\section{Acknowledgements}

2 This work was supported by a Wellcome Trust - NIH PhD studentship (200934/Z/16/Z) to A.M.;

3 a National Institute for Health Research (NIHR) Biomedical Research Center (BRC) fellowship to

4 V.V.; and by a Wellcome Investigator Award (101798/Z/13/Z) to J.P.R. The funders had no role

5 in the study design, data collection and analysis, decision to publish or preparation of the

6 manuscript. For the purpose of Open Access, the author has applied a CC BY public copyright

7 licence to any Author Accepted Manuscript version arising from this submission. The authors

8 thank Eoin Travers and Oliver J. Robinson for valuable input on an earlier version of the

9 manuscript. A preprint was previously published at bioRxiv

10 (https://doi.org/10.1101/2021.06.30.450026).

\section{Competing interests}

13 The authors have no competing interests to declare.

\section{Authors' contribution}

16 A.M. and J.P.R. conceived and designed the study. A.M. acquired the data and performed

17 analyses under the supervision of V.V. and J.P.R. All authors contributed to interpreting the

18 data and drafting or substantially revising of the manuscript. 


\section{References}

2 Adams, R. A., Huys, Q. J., \& Roiser, J. P. (2016). Computational Psychiatry: towards a mathematically informed understanding of mental illness. J Neurol Neurosurg Psychiatry, 87(1), 53-63. doi:10.1136/jnnp-2015-310737

Ahn, W. Y., \& Busemeyer, J. R. (2016). Challenges and promises for translating computational tools into clinical practice. Curr Opin Behav Sci, 11, 1-7. doi:10.1016/j.cobeha.2016.02.001

Ahn, W. Y., Haines, N., \& Zhang, L. (2017). Revealing Neurocomputational Mechanisms of Reinforcement Learning and Decision-Making With the hBayesDM Package. Comput

Aylward, J., Valton, V., Ahn, W. Y., Bond, R. L., Dayan, P., Roiser, J. P., \& Robinson, O. J. (2019). Altered learning under uncertainty in unmedicated mood and anxiety disorders. Nat Hum Behav, 3, 1116-1123. doi:10.1038/s41562-019-0628-0

Baek, K., Kwon, J., Chae, J. H., Chung, Y. A., Kralik, J. D., Min, J. A., . . Jeong, J. (2017). Heightened aversion to risk and loss in depressed patients with a suicide attempt Elliott, R. (2016). EMOTICOM: A Neuropsychological Test Battery to Evaluate Emotion, Motivation, Impulsivity, and Social Cognition. Front Behav Neurosci, 10, 25. doi:10.3389/fnbeh.2016.00025

21 Brown, J. K., Waltz, J. A., Strauss, G. P., McMahon, R. P., Frank, M. J., \& Gold, J. M. (2013). Hypothetical decision making in schizophrenia: the role of expected value computation 
and "irrational" biases. Psychiatry Res, 209(2), 142-149.

Brown, V. M., Chen, J., Gillan, C. M., \& Price, R. B. (2020). Improving the Reliability of Computational Analyses: Model-Based Planning and Its Relationship With Compulsivity. Biol Psychiatry Cogn Neurosci Neuroimaging, 5(6), 601-609. doi:10.1016/j.bpsc.2019.12.019

Browning, M., Behrens, T. E., Jocham, G., O'Reilly, J. X., \& Bishop, S. J. (2015). Anxious

Browning, M., Carter, C. S., Chatham, C., Den Ouden, H., Gillan, C. M., Baker, J. T., ... Paulus, M. (2020). Realizing the Clinical Potential of Computational Psychiatry: Report From the Banbury Center Meeting, February 2019. Biol Psychiatry, 88(2), e5-e10. doi:10.1016/j.biopsych.2019.12.026

17 Charpentier, C. J., Aylward, J., Roiser, J. P., \& Robinson, O. J. (2017). Enhanced Risk Aversion, 1014-1022. doi:10.1016/j.biopsych.2016.12.010 loss aversion and striatal-amygdala coupling in low-anxious individuals. Soc Cogn Affect Neurosci, 11(4), 569-579. doi:10.1093/scan/nsv139 
1 Charpentier, C. J., De Neve, J. E., Li, X., Roiser, J. P., \& Sharot, T. (2016). Models of Affective Decision Making: How Do Feelings Predict Choice? Psychol Sci, 27(6), 763-775. doi:10.1177/0956797616634654

Chung, D., Kadlec, K., Aimone, J. A., McCurry, K., King-Casas, B., \& Chiu, P. H. (2017). Valuation in major depression is intact and stable in a non-learning environment. Sci Rep, 7, 44374. doi:10.1038/srep44374

Daw, N. D. (2011). Trial-by-trial data analysis using computational models. In Decision Making, Affect, and Learning: Attention and Performance XXIII: Oxford University Press.

Daw, N. D., \& Doya, K. (2006). The computational neurobiology of learning and reward. Curr Opin Neurobiol, 16(2), 199-204. doi:10.1016/j.conb.2006.03.006

Daw, N. D., O'Doherty, J. P., Dayan, P., Seymour, B., \& Dolan, R. J. (2006). Cortical substrates for Neurobiol, 18(2), 185-196. doi:10.1016/j.conb.2008.08.003 American Psychiatric Association. 
New Empirical Evidence and a Meta-Analysis. Psychol Sci, 31(7), 792-806. doi:10.1177/0956797620916786

3

Enkavi, A. Z., Eisenberg, I. W., Bissett, P. G., Mazza, G. L., MacKinnon, D. P., Marsch, L. A., \& Poldrack, R. A. (2019). Large-scale analysis of test-retest reliabilities of self-regulation measures. Proc Natl Acad Sci U S A, 116(12), 5472-5477. doi:10.1073/pnas.1818430116

Eshel, N., \& Roiser, J. P. (2010). Reward and punishment processing in depression. Biol Psychiatry, 68(2), 118-124. doi:10.1016/j.biopsych.2010.01.027

Faul, F., Erdfelder, E., Lang, A. G., \& Buchner, A. (2007). G*Power 3: a flexible statistical power analysis program for the social, behavioral, and biomedical sciences. Behav Res Methods, 39(2), 175-191. doi:10.3758/bf03193146

Fleiss, J. L. (2011). Reliability of Measurement. In The Design and Analysis of Clinical Experiments (pp. 1-32).

Frey, R., Pedroni, A., Mata, R., Rieskamp, J., \& Hertwig, R. (2017). Risk preference shares the psychometric structure of major psychological traits. Sci Adv, 3(10), e1701381. doi:10.1126/sciadv.1701381

Friston, K. J., Redish, A. D., \& Gordon, J. A. (2017). Computational Nosology and Precision Psychiatry. Comput Psychiatr, 1, 2-23. doi:10.1162/CPSY_a_00001

Gillan, C. M., Kosinski, M., Whelan, R., Phelps, E. A., \& Daw, N. D. (2016). Characterizing a psychiatric symptom dimension related to deficits in goal-directed control. Elife, 5. doi:10.7554/eLife.11305 
1 Glockner, A., \& Pachur, T. (2012). Cognitive models of risky choice: parameter stability and predictive accuracy of prospect theory. Cognition, 123(1), 21-32. doi:10.1016/j.cognition.2011.12.002

Hadlaczky, G., Hokby, S., Mkrtchian, A., Wasserman, D., Balazs, J., Machin, N., . . . Carli, V.

Haines, N., Kvam, P., Irving, L., Smith, C., Beauchaine, T. P., Pitt, M. A., . . Turner, B. (2020). Learning from the Reliability Paradox: How Theoretically Informed Generative Models Can Advance the Social, Behavioral, and Brain Sciences. PsyArXiv.

Hartley, C. A., \& Phelps, E. A. (2012). Anxiety and decision-making. Biol Psychiatry, 72(2), 113118. doi:10.1016/j.biopsych.2011.12.027

Hedge, C., Powell, G., \& Sumner, P. (2018). The reliability paradox: Why robust cognitive tasks do not produce reliable individual differences. Behav Res Methods, 50(3), 1166-1186.

Huys, Q. J. M. (2018). Advancing Clinical Improvements for Patients Using the Theory-Driven and Data-Driven Branches of Computational Psychiatry. JAMA Psychiatry, 75(3), 225computational understanding of mental illness. Neuropsychopharmacology, 46(1), 3-19. 
1 Huys, Q. J. M., Maia, T. V., \& Frank, M. J. (2016). Computational psychiatry as a bridge from neuroscience to clinical applications. Nat Neurosci, 19(3), 404-413. doi:10.1038/nn.4238

Huys, Q. J. M., Moutoussis, M., \& Williams, J. (2011). Are computational models of any use to psychiatry? Neural Netw, 24(6), 544-551. doi:10.1016/j.neunet.2011.03.001

Kahneman, D., \& Tversky, A. (1979). Prospect Theory: An Analysis of Decision under Risk.

7 Klaus, F., Chumbley, J. R., Seifritz, E., Kaiser, S., \& Hartmann-Riemer, M. (2020). Loss Aversion and Risk Aversion in Non-Clinical Negative Symptoms and Hypomania. Front Psychiatry,

10 Koo, T. K., \& Li, M. Y. (2016). A Guideline of Selecting and Reporting Intraclass Correlation Coefficients for Reliability Research. J Chiropr Med, 15(2), 155-163. doi:10.1016/j.jcm.2016.02.012

13 Kruschke, J. K. (2015). Doing Bayesian Data Analysis: A tutorial with R, JAGS, and Stan (Second ed.). Amsterdam: Academic Press.

Lakens, D. (2013). Calculating and reporting effect sizes to facilitate cumulative science: a practical primer for t-tests and ANOVAs. Frontiers in Psychology, 4(863), 863. doi:10.3389/fpsyg.2013.00863

Maia, T. V. (2009). Reinforcement learning, conditioning, and the brain: Successes and challenges. Cogn Affect Behav Neurosci, 9(4), 343-364. doi:10.3758/CABN.9.4.343 neurological disorders. Nat Neurosci, 14(2), 154-162. doi:10.1038/nn.2723 
1 McGraw, K. O., \& Wong, S. P. (1996). Forming inferences about some intraclass correlation coefficients. Psychological Methods, 1(1), 30-46. doi:10.1037/1082-989x.1.1.30

Mkrtchian, A., Aylward, J., Dayan, P., Roiser, J. P., \& Robinson, O. J. (2017). Modeling Avoidance in Mood and Anxiety Disorders Using Reinforcement Learning. Biol Psychiatry, 82(7), 532-539. doi:10.1016/j.biopsych.2017.01.017

Mkrtchian, A., Roiser, J. P., \& Robinson, O. J. (2017). Threat of shock and aversive inhibition:

Montague, P. R., Dolan, R. J., Friston, K. J., \& Dayan, P. (2012). Computational psychiatry. Trends Cogn Sci, 16(1), 72-80. doi:10.1016/j.tics.2011.11.018

Montague, P. R., Hyman, S. E., \& Cohen, J. D. (2004). Computational roles for dopamine in behavioural control. Nature, 431(7010), 760-767. doi:10.1038/nature03015

Moutoussis, M., Bullmore, E. T., Goodyer, I. M., Fonagy, P., Jones, P. B., Dolan, R. J., . . .

17 Nair, A., Rutledge, R. B., \& Mason, L. (2020). Under the Hood: Using Computational Psychiatry to Make Psychological Therapies More Mechanism-Focused. Front Psychiatry, 11, 140. 
1 Noble, S., Scheinost, D., \& Constable, R. T. (2019). A decade of test-retest reliability of functional connectivity: A systematic review and meta-analysis. Neuroimage, 203, 116157. doi:10.1016/j.neuroimage.2019.116157

Nord, C. L., Gray, A., Charpentier, C. J., Robinson, O. J., \& Roiser, J. P. (2017). Unreliability of putative fMRI biomarkers during emotional face processing. Neuroimage, 156, 119-127. doi:10.1016/j.neuroimage.2017.05.024

Palminteri, S., \& Chevallier, C. (2018). Can We Infer Inter-Individual Differences in Risk-Taking From Behavioral Tasks? Front Psychol, 9, 2307. doi:10.3389/fpsyg.2018.02307

Palminteri, S., Wyart, V., \& Koechlin, E. (2017). The Importance of Falsification in Computational Cognitive Modeling. Trends Cogn Sci, 21(6), 425-433. doi:10.1016/j.tics.2017.03.011

Patzelt, E. H., Hartley, C. A., \& Gershman, S. J. (2018). Computational Phenotyping: Using Models to Understand Individual Differences in Personality, Development, and Mental Illness. Personal Neurosci, 1, e18. doi:10.1017/pen.2018.14

Paulus, M. P., Huys, Q. J., \& Maia, T. V. (2016). A Roadmap for the Development of Applied Computational Psychiatry. Biol Psychiatry Cogn Neurosci Neuroimaging, 1(5), 386-392. doi:10.1016/j.bpsc.2016.05.001

Paulus, M. P., \& Thompson, W. K. (2019). Computational approaches and machine learning for individual-level treatment predictions. Psychopharmacology (Berl). doi:10.1007/s00213019-05282-4

Plichta, M. M., Schwarz, A. J., Grimm, O., Morgen, K., Mier, D., Haddad, L., . . MeyerLindenberg, A. (2012). Test-retest reliability of evoked BOLD signals from a cognitive- 
emotive fMRI test battery. Neuroimage, 60(3), 1746-1758.

Price, R. B., Brown, V., \& Siegle, G. J. (2019). Computational Modeling Applied to the Dot-Probe Task Yields Improved Reliability and Mechanistic Insights. Biol Psychiatry, 85(7), 606612. doi:10.1016/j.biopsych.2018.09.022

Robinson, O. J., \& Chase, H. W. (2017). Learning and Choice in Mood Disorders: Searching for the Computational Parameters of Anhedonia. Comput Psychiatr, 1(1), 208-233.

Rodebaugh, T. L., Scullin, R. B., Langer, J. K., Dixon, D. J., Huppert, J. D., Bernstein, A., . . Lenze, E. J. (2016). Unreliability as a threat to understanding psychopathology: The cautionary

12 Ruggeri, K., Ali, S., Berge, M. L., Bertoldo, G., Bjorndal, L. D., Cortijos-Bernabeu, A., ... Folke, T. (2020). Replicating patterns of prospect theory for decision under risk. Nat Hum Behav,

Scheibehenne, B., \& Pachur, T. (2015). Using Bayesian hierarchical parameter estimation to assess the generalizability of cognitive models of choice. Psychon Bull Rev, 22(2), 391407. doi:10.3758/s13423-014-0684-4 naturalistic risk-taking with cognitive neuroscience. Trends Cogn Sci, 15(1), 11-19. doi:10.1016/j.tics.2010.10.002 
1 Seymour, B., Daw, N. D., Roiser, J. P., Dayan, P., \& Dolan, R. (2012). Serotonin selectively modulates reward value in human decision-making. J Neurosci, 32(17), 5833-5842. doi:10.1523/JNEUROSCI.0053-12.2012

Shahar, N., Hauser, T. U., Moutoussis, M., Moran, R., Keramati, M., consortium, N., \& Dolan, R. J. (2019). Improving the reliability of model-based decision-making estimates in the twostage decision task with reaction-times and drift-diffusion modeling. PLoS Comput Biol, 15(2), e1006803. doi:10.1371/journal.pcbi.1006803

Sip, K. E., Gonzalez, R., Taylor, S. F., \& Stern, E. R. (2017). Increased Loss Aversion in Unmedicated Patients with Obsessive-Compulsive Disorder. Front Psychiatry, 8, 309.

11 Sokol-Hessner, P., Hsu, M., Curley, N. G., Delgado, M. R., Camerer, C. F., \& Phelps, E. A. (2009). Thinking like a trader selectively reduces individuals' loss aversion. Proc Natl Acad Sci U S A, 106(13), 5035-5040. doi:10.1073/pnas.0806761106

14 Sokol-Hessner, P., \& Rutledge, R. B. (2019). The Psychological and Neural Basis of Loss Aversion. Current Directions in Psychological Science, 28(1), 20-27. doi:10.1177/0963721418806510

17 Speekenbrink, M., \& Konstantinidis, E. (2015). Uncertainty and exploration in a restless bandit problem. Top Cogn Sci, 7(2), 351-367. doi:10.1111/tops.12145 
1 Teufel, C., \& Fletcher, P. C. (2016). The promises and pitfalls of applying computational models to neurological and psychiatric disorders. Brain, 139(Pt 10), 2600-2608. doi:10.1093/brain/aww209

Tremeau, F., Brady, M., Saccente, E., Moreno, A., Epstein, H., Citrome, L., . . J Javitt, D. (2008). Loss aversion in schizophrenia. Schizophr Res, 103(1-3), 121-128. doi:10.1016/j.schres.2008.03.027

7 Tversky, A., \& Kahneman, D. (1992). Advances in Prospect Theory: Cumulative Representation of Uncertainty. Journal of Risk and Uncertainty, 5(4), 297-323. doi:Doi

Valton, V., Wise, T., \& Robinson, O. J. (2020). Recommendations for Bayesian hierarchical model specifications for case-control studies in mental health. Machine Learning for Health (ML4H) at NeurIPS 2020, 34th Conference on Neural Information Processing Systems. arXiv:2011.01725 [cs.CY] doi:http://arxiv.org/abs/2011.01725

14 Wang, X. J., \& Krystal, J. H. (2014). Computational psychiatry. Neuron, 84(3), 638-654. doi:10.1016/j.neuron.2014.10.018

Wiecki, T. V., Poland, J., \& Frank, M. J. (2015). Model-Based Cognitive Neuroscience Approaches to Computational Psychiatry. Clinical Psychological Science, 3(3), 378-399. doi:10.1177/2167702614565359

19 Wilson, R. C., \& Collins, A. G. (2019). Ten simple rules for the computational modeling of behavioral data. Elife, 8. doi:10.7554/eLife.49547

21 Yi, M. S. K., Steyvers, M., \& Lee, M. (2009). Modeling Human Performance in Restless Bandits 
bioRxiv preprint doi: https://doi.org/10.1101/2021.06.30.450026; this version posted November 7, 2021. The copyright holder for this preprint (which was not certified by peer review) is the author/funder, who has granted bioRxiv a license to display the preprint in perpetuity. It is made available under aCC-BY 4.0 International license.

1 